\title{
Criminologie
}

\section{Adolescent et agresseur sexuel : bilan d'une recherche}

\section{Jean Dozois}

Volume 27, numéro 2, 1994

Sexe et criminalité

URI : https://id.erudit.org/iderudit/017356ar

DOI : https://doi.org/10.7202/017356ar

Aller au sommaire du numéro

Éditeur(s)

Les Presses de l'Université de Montréal

ISSN

0316-0041 (imprimé)

1492-1367 (numérique)

Découvrir la revue

Citer cet article

Dozois, J. (1994). Adolescent et agresseur sexuel : bilan d'une recherche.

Criminologie, 27(2), 71-85. https://doi.org/10.7202/017356ar

\section{Résumé de l'article}

The purpose of this research project was to gain a better knowledge of juvenile sex offenders in the Montreal area. Do they present differences or similarities with other subjects from studies made in the United States? Can we improve our assessment techniques to better differentiate those who should be treated in a closed setting from those who could benefit from a follow-up in the community? And how does our juvenile justice system deal with this type of offender? In order to find answers to these issues, ten (10) in-depth interviews with personality measures were conducted with juveniles who admitted (or were convicted of) sexual abuse. Moreover, a study of fifty (50) files from the Social Services relating to the same kind of behavior was done in the Montreal area. Our results are similar to other studies made elsewhere : those who where convicted of rape or child molesting committed their first (official) offense at a mean age of 14.5 years and $60 \%$ of their victims were females of an average age of 9 . For $38 \%$ of our sample, the sexual offense is part of an heterogenous criminal career. Our personnality measures failed to differentiate between child molesters and rapists. However, on the Jesness Inventory, our ten subjects had high scores on the SM (Social Maladjustment) scale, and on AI (Asocial Index). One other significant finding was that the personnel involved with assessment and treatment of juvenile sex offenders had to get a better knowledge of the dynamics involved with such offenses to record basic information (victim's age, the exact nature of the behavior and the type of violence involved) and recommend appropriate treatment.
Ce document est protégé par la loi sur le droit d'auteur. L’utilisation des services d'Érudit (y compris la reproduction) est assujettie à sa politique d'utilisation que vous pouvez consulter en ligne.

https://apropos.erudit.org/fr/usagers/politique-dutilisation/ 
The purpose of this research project was to gain a better knowledge of juvenile sex offenders in the Montreal area. Do they present differences or similarities with other subjects from studies made in the United States? Can we improve our assessment techniques to better differentiate those who should be treated in a closed setring from those who could benefit from a follow-up in the community? And how does our juvenile justice system deal with this type of offender? In order to find answers to these issues, ten (10) in-depth interviews with personality measures were conducted with juveniles who admitted (or were convicted of) sexual abuse. Moreover, a study of fifty (50) files from the Social Services relating to the same kind of behavior was done in the Montreal area. Our results are similar to other studies made elsewhere: those who where convicted of rape or child molesting committed their first (official) offense at a mean age of 14.5 years and $60 \%$ of their victims were females of an average age of 9 . For $38 \%$ of our sample, the sexual offense is part of an heterogenous criminal career. Our personnality measures failed to differentiate between child molesters and rapists. However, on the Jesness Inventory, our ten subjects had high scores on the SM (Social Maladjustment) scale, and on AI (Asocial Index). One other significant finding was that the personnel involved with assessment and treatment of juvenile sex offenders had to get a better knowledge of the dynamics involved with such offenses to record basic information (victim's age, the exact nature of the behavior and the type of violence involved) and recommend appropriate treatment.

\section{INTRODUCTION}

Cet article traitant de la violence sexuelle commise par des adolescents s'inspire largement d'un rapport de recherche ${ }^{2}$ publié en juin 1993 en

1. Professeur à l'École de criminologie de l'Universtié de Montréal, C.P. 6128, Succ. A, Montréal, Québec, H3C 337.

2. Cette recherche a ćté rendue possible grâce à une subvention de la Donation Richelieu et le rapport est disponible au Centre international de criminologic comparéc de l'Université de Montréal. 
collaboration avec Yves Boisvert, Denis Lafortune et Diane Maisonneuve ${ }^{3}$. L'auteur désire donc exprimer sa reconnaissance à ces personnes qui ont alimenté, par leurs commentaires, la réflexion qui a conduit à l'élaboration de cet article.

En une vingtaine d'années. l'état des connaissances sur les principaux types d'abus sexuels. qu'il s'agisse du viol, de la pédophilie ou de l'inceste. a progressé de façon décisive. Mais il demeure que la plupart des développements importants sont d'abord apparus du côté de la «victimologie». En effet. bon nombre de travaux réalisés dans les années 1970 et 1980 ont permis de reconnaître les répercussions à court. à moyen et à long terme que peuvent avoir les délits sexuels sur les enfants, adolescents ou adultes qui en ont été victimes. Les travaux de Burgess (1978), Kempe et Kempe(1978) et Sgroi (1981) ont vite pris de la notoriété. Pendant ce temps, l'étude clinique des auteurs d'agressions sexuelles met plus de temps à se structurer. Lentement, les contributions de Gebhard et al. (1965) et de Groth et Birnbaum (1979) viennent éclairer la question des motivations avouées et inavouées des abuseurs sexuels adultes. Celles de Shoor t al. (1962) et de Lewis et al. (1979) explorent le monde des abuseurs adolescents. Mais la plus grande part des écrits reste consacrée aux victimes et aux séquelles liées aux abus.

Au Québec, la situation peut être qualifiée de similaire. En ce qui concerne le Comité de la protection de la jeunesse, Manseau (1990) constate que depuis 1979 , ce comité a permis la réalisation d'études majeures sur les abus sexuels, alors que des problèmes tout aussi importants que la mendicité, l'absentéisme scolaire ou les fugues n'ont fait l'objet d'aucune étude approfondie. Comme aux États-Unis, l'intêrêt porté aux abus sexuels s'est accompagné d'une insistance sur les jeunes victimes. Ces garçons et ces fillettes, dont la sécurité et le développement étaient considérés comme compromis, sont alors apparus au centre des interventions et des efforts de réflexion.

Ce n'est que plus récemment que les Beltrami et al. (1979), Aubut et Lamontagne (1980) et Van Gijseghem (1988) se sont véritablement penchés sur la personnalité de l'abuseur sexuel.

S'il est une problématique autour de laquelle les réflexions et projets d'interventions se font discrets, c'est bien celle des adolescents ayant commis un geste sexuel abusif. Du côté des écrits québécois. un ouvrage de Szabo (1960) intitulé Contribution à l'étude de la délinquance sexuelle a

3. Yves Boisvert, psycho-éducateur.

Denis Lafortune, psychologuc à l'Unité des adolescents du Pavillon Albert-Prévost.

Diane Maisonneuve, criminologue au Service jeunes contrevenants, praticienne du CPEJ du Montréal-centre. 
effleuré le sujet. Nous disons «effleuré», parce qu'à cette époque l'on entendait surtout par «délinquance sexuelle juvénile» la prostitution de mineur(e)s qui représentait plus de $78 \%$ des délits retenus dans son échantillon. De nos jours, il est plus difficile de regrouper, sous un même terme, les auteurs de gestes abusifs et les prostitué(e)s. En effet. lors de l'adoption de la Loi sur la protection de la jeunesse, l'accent mis sur la protection des mineur(e)s a fait passer les jeunes faisant de la prostitution du côté de la victimologie.

\section{LA VALIDITÉ CONTROVERSÉE DES STATISTIQUES OFFICIELLES}

L'incidence de toute forme d'abus sexuel est statistiquement fort difficile à établir avec précision. En effet, depuis quelques années, la valeur de ces statistiques criminelles est fortement critiquée. D'un côté, l'on parle d'un discours «alarmiste» : après avoir étudié attentivement les statistiques du Comité de la protection de la jeunesse, Manseau (1990) souligne combien ces documents mêlaient à des cas d'abus «jugés fondés » des signalements «ni vérifiés, ni vérifiables». gonflant ainsi les chiffres. De plus, elle a avancé que cette hausse des signalements pouvait être partiellement attribuée aux changements terminologiques apportés aux lois. en 1979 et en 1984. En revanche, certains ont affirmé que les statistiques officielles sousestimaient la réalité. Plusieurs personnes ont tenté de démontrer que l'abus sexuel était l'un des crimes les plus sous-rapportés et que présentement l'on ne voyait que la pointe de l'iceberg en matière de délinquance sexuelle.

Pour arriver à se situer face à l'importance de ces signalements, notre groupe en est venu à se représenter l'enregistrement d'un cas d'abus sexuel comme le fruit de deux types de décisions. Le premier type concerne celle prise par les victimes. Il est généralement admis qu'une victime sur cinq dénonce son agresseur aux autorités (Knight et al.. 1985). Nous nous situons donc du côté de ceux qui croient que plus souvent qu'autrement, c'est la loi du silence qui prévaut. De plus, nous affirmons que différents facteurs, tels que le degré de génitalité de l'acte (attouchement. fellation ou pénétration) ou le degré de familiarité avec l'agresseur (famille, connaissance, étranger), peuvent influencer le choix qui sera fait, par l'enfant, l'adolescent ou l'adulte abusé, de dénoncer ou non son agresseur.

Le deuxième type de décisions est en rapport avec l'adulte. le parent, le praticien ou le représentant de la loi, qui entend les allégations de la victime ou les aveux de l'agresseur. Cet adulte aura à décider s'il prête foi aux propos qu'il a entendus. S'il est un intervenant professionnel, déciderat-il de signaler le cas aux autorités, comme la loi l'exige, ou restera-t-il fidèle à une longue tradition de secret professionnel ? Et s'il signale le cas d'un agresseur adolescent aux autorités, le tera-t-il en fonction de l'article 
$38 \mathrm{~h}$ ) de la Loi de la protection de la jeunesse. ou se tournera-t-il plutôt vers la Loi sur les jeunes contrevenants?

\section{LA MULTIDISCIPLINARITÉ DU CHAMP}

Un constat s'impose : l'intervention auprès des auteurs d'agression sexuelle intéresse plusieurs disciplines. L'abus sexuel est un large secteur d'activités auquel s'intéressent les psychiatres, psychologues. psychoéducateurs, criminologues, avocats, sexologues et travailleurs sociaux. Chacun amenant avec lui sa formation universitaire et ses références théoriques, il s'ensuit un enchevêtrement de réflexions et de pratiques diverses. En effet, l'étude des agresseurs sexuels pourrait être entreprise sous plusieurs angles théoriques allant de la victimologie aux approches cognitivecomportementale. systémique/familiale, sociologique, psychodynamique, etc.

Quoi qu'il en soit, ce n'est que récemment. au Québec, que l'on tente d'apporter un certain éclairage à cette problématique des agressions sexuelles commises par des adolescents. Comme pour tout nouvel intérêt, les énergies au début sont très éparpillées et ne procèdent d'aucune concertation. La planification de l'intervention repose plus sur des déductions faites à partir d'études réalisées aux États-Unis ou en Ontario que sur une observation systérnatique de cas concrets en provenance de programmes d'intervention québécois.

Chacune des disciplines analyse la problématique de l'agression sexuelle sous un angle qui lui est propre. Par exemple, les professionnels ceuvrant auprès de délinquants sexuels adultes pourraient être tentés d'aborder le phénomène de la même façon avec les adolescents : judiciariser tous les cas d'agression, les évaluer et les traiter selon des méthodes développées avec les adultes. Pour certains jeunes présentant plusieurs caractéristiques des délinquants sexuels adultes, et comportant un fort potentiel de risque, ce sera sûrement une bonne chose. Pour d'autres, chez qui il n'y a pas de précocité au niveau de l'intérêt sexuel déviant ni de précocité dans les comportements, les professionnels pourraient présumer qu'il y a peu de risque que s'établisse un «pattern» de comportement associé à une délinquance sexuelle adulte potentielle. Selon Barbaree, Marshall et Hudson (1993). les adolescents ayant commis une agression sexuelle ne peuvent être automatiquement assimilés à des délinquants adultes qui seraient tout juste un peu plus jeunes. D' autre part. certains intervenants du réseau juvénile, travaillant surtout avec des jeunes présentant une problématique de mésadaptation sociale. pourraient considérer que ce type d'agir n'est qu'une nouvelle manifestation d'un syndrome plus global (des «troubles de comportement sérieux ») et, en vertu d'une approche psycho-sociale, déju- 
diciariser la situation toutes les fois où c'est possible et intervenir en fonction de cette approche. Encore là, pour certains adolescents. ce pourrait être tout à fait approprié alors que pour d'autres. ce ne serait pas le cas.

Les statistiques et la pratique révèlent que les cas d'agressions sexuelles commises par les adolescents sont de plus en plus nombreux. D'autre part. s'il existe certaines études visant à préciser le chiffre noir des agressions sexuelles commises par des adultes (Abel, Mittelman. Becker, 1984). peu d'écrits concernent les infractions sexuelles commises par les adolescents.

Les résultats de plusieurs recherches semblent toutefois converger vers des résultats similaires. Tout d'abord, aux États-Unis, une proportion importante de l'ensemble des agressions sexuelles est commise par des adolescents : approximativement $20 \%$ des tous les viols et de 30 à $50 \%$ des gestes pédophiliques sont le fait d'adolescents (Becker. Kaplan. Cunningham-Rathner et Kavoussi 1986). En second lieu. ces mêmes auteurs travaillant auprès de délinquants sexuels adultes estiment généralement que $50 \%$ de leur clientèle ont amorcé leur carrière à l'adolescence. Dans le but de mieux cerner cette problématique au Québec, nous avons structuré un projet de recherche s'adressant directement à cette population, avec l'espoir que les résultats nous permettront de mettre sur pied des programmes d'évaluation et d'intervention plus spécifiques.

Formé en 1989, le Comité d'étude sur les jeunes agresseurs sexuels (CEJAS) s'est proposé de mener une étude descriptive auprès d'adolescents québécois ayant commis un abus sexuel.

Face à l'absence de consensus théorique. deux voies ont été envisagées. D'une part, l'on pouvait tenter de régler les débats en formulant de nouvelles approches-synthèses qui rallieraient tous les partis. Cette première option présentait toutefois le risque de mener à des bilans schématiques. négligeant parfois l'incompatibilité des positions en présence. D'autre part. l'on pouvait se retrancher derrière un certain nombre d'observations empiriques et opérationnalisables. C'est ce deuxième choix que nous avons privilégié afin d'augmenter les possibilités d'échanges, de comparaisons et de réplications de l'étude, quitte à perdre un peu de profondeur clinique. Nous avons voulu dégager les antécédents socio-tamiliaux de ces adolescents, certains traits de personnalité qu'ils pouvaient avoir en commun, établir différents profils types d'adolescents abuseurs et identifier des priorités en termes de développement de programmes d'intervention.

L'étude exploratoire repose d'abord sur dix (10) entrevues en profondeur réalisées auprès d'adolescents ayant participé à une forme d'abus sexuel selon l'un des critères suivants : 1) avoir reconnu la matérialité des faits dans le cadre d'une mesure de rechange prévue à la Loi sur les jeunes contrevenants: 2) avoir été déclaré coupable ou avoir plaidé coupable en 
vertu de cette même loi ; 3 ) avoir été signalé pour «troubles de comportement sérieux» en vertu de la Loi sur la protection de la jeunesse. Ces entrevues en profondeur comportaient une grille d'entrevue semi-structurée. en quatre mesures de personnalité ainsi qu'une mesure des capacités intellectuelles.

D'autre part. nous avons également effectué une étude portant sur cinquante (50) dossiers d'adolescents de CSS ou placés en centre de réadaptation dans la région de Montréal.

Ces dossiers avaient été choisis en fonction des mêmes critères énoncés précédemment et nous avons décidé de limiter notre analyse aux variables liées à l'agir délictuel ainsi qu'aux caractéristiques des victimes.

\section{ANALYSE DES RÉSULTATS OBTENUS SUITE AUX ENTREVUES SEMI-STRUCTURÉES}

Notre échantillon de 10 sujets présentait un âge moyen de 13.9 ans lors des gestes reprochés. La moitié tombaient sous la Loi des jeunes contrevenants et la moitié sous la Loi sur la protection de la jeunesse. Ils en étaient tous à leur première comparution pour un geste de nature sexuelle. mais la majorité d'entre eux $(70 \%)$ avaient des antécédents de délinquance autre que sexuelle. Tous avaient reconnu leur culpabilité à un délit de nature sexuelle (L.J.C.) ou reconnaissaient avoir posé un geste à connotation sexuelle dans le cadre de l'article $38 \mathrm{~h}$ ) de la L.P.J. (troubles de comportement sérieux).

Au plan de l'agir, la majorité des gestes reprochés sont des attouchements $(60 \%)$ commis avec une violence minimale, généralement sans complice $(80 \%)$, sur une victime de sexe féminin, dont l'âge moyen était de 6 ans et connue du sujet dans $90 \%$ des cas. Il s'agit rarement d'un événement unique, les gestes se répétant et se prolongeant sur plusieurs mois avant qu'une plainte soit portée.

Au plan de la reconnaissance du délit, la majorité de nos sujets (9/10), reconnaissent avoir posé les gestes qu'on leur reproche. Cependant. à peine $4 / 10$ sont conscients du caractère délictuel de leur geste et reconnaissent avoir un problème à ce niveau (note : 3 de ces 4 sujets avaient déjà participé à une thérapie pour jeunes abuseurs sexuels). La moitié de nos sujets rationalisent ou minimisent l'importance du geste posé. Sept sujets sur 10 croyaient leur victime consentante au moment de l'initiation du délit (ou même sollicitante dans certains cas). Trois sujets sur 10 reconnaissaient avoir une fantasmatique déviante reliée au délit, fantasmatique qui peut être utilisée lors d'activités masturbatoires. Enfin, 3/10 avouent une certaine planification du délit. 
Quant à leur milieu social, la majorité des sujets proviennent de familles dont le niveau socio-économique est moyen $(40 \%)$ ou défavorisé $(50 \%)$. Six d'entre eux ont vécu au moins un placement extra-familial et ont été exposés à des modèles de violence physique $(60 \%)$ ou des modèles sexuels inadéquats $(30 \%)$. La majorité de nos sujets ont vécu des difficultés relationnelles avec leurs parents, ont eu des difficultés au niveau scolaire, n'ont pas de réseau social (amis) minimal et ont eux-mêmes été victimes d'abus physiques ou sexuels.

Au Jesness. les 10 sujets obtiennent un score supérieur à 60 à l'échelle de mésapdaptation sociale (SM) et à l'indice d'asocialité. Certains (8/10) obtiennent un score supérieur à 55 pour l'orientation aux valeurs (VO), $7 / 10$ obtiennent un score supérieur à 55 pour l'agressivité manifeste (MA) et $6 / 10$ obtiennent un score supérieur à 55 pour l'autisme (AU).

Les résultats aux tests psychométriques (matrice de Raven. échelle de Nowicki et Strickland sur le lieu de contrôle interne ou externe, échelle de Raskin et Hall sur la personnalité narcissique et échelle de Novaco sur le contrôle de la colère) ne présentent pas de différence significative entre les sujets ayant commis des attouchements et ceux ayant commis ou tenté de commettre un viol. Les sujets se situent généralement dans la normale pour leur performance aux quatre tests utilisés. On aurait pu s'attendre à des écarts plus importants entre ces deux groupes (Boisvert, 1990), mais il faut tenir compte du petit nombre de sujets de notre échantillon. Éventuellement, il pourrait être intéressant de comparer les résultats obtenus à ces tests avec une clientèle plus importante.

\section{ANALYSE DES RÉSULTATS OBTENUS SUITE À LA CONSULTATION DE DOSSIERS PSYCHO-SOCIAUX}

Dans l'espoir d'obtenir un portrait plus exhaustif de la situation de ces adolescents, les chercheurs ont entrepris un étude de $\mathbf{5 0}$ nouveaux dossiers. Ces dossiers ont été étudiés et consultés dans les bureaux des centres de services sociaux ou des centres de réadaptation. Certains dossiers appartenaient au service de la protection de la jeunesse (7) et la majorité au service des jeunes contrevenants (43). Le nombre total des dossiers consultés se répartit de la façon suivante : CSSMM : 22, CSSLL : 13, CSSR : 10, Cité des Prairies : 2, Mont-Saint-Antoine : 3 dossiers.

Les variables retenues lors de la consultation des dossiers psychosociaux ne concernent que certaines caractéristiques criminologiques: celles reliées aux données légales (les mesures prises à l'endroit des sujets et les antécédents de délinquance officiels ou non officiels): celles concernant les données liées à l'agir (l'âge de l'adolescent lors de la commission du délit. lá nature des actes posés et le niveau de violence associée); celles 
liêes à la victime (le nombre, l'âge, le sexe ainsi que la nature du lien entre l'agresseur et la victime).

Compte tenu de la disparité des informations disponibles sur certaines caractéristiques, nous avons choisi d'éliminer celles-ci : le niveau de reconnaissance du délit, les données socio-familiales et les données développementales. De plus, lorsqu'il a été question de regrouper certaines caractéristiques de l'étude. nous avons fait le constat suivant : seulement 36 dossiers sur $\mathbf{5 0}$ comprenaient des informations sur toutes les variables concernées.

\section{A) Données légales}

Les mesures prises en fonction de la Loi sur les jeunes contrevenants concernent 43 sujets de l'échantillon. Sept d'entre eux ont bénéficié d'une mesure de rechange: sept autres sujets ont obtenu une ordonnance de mise sous garde (4 mises sous garde ouverte et 3 mises sous garde fermée) et finalement. vingt-neuf sujets ont obtenu une ordonnance de probation.

Les sept autres sujets sont sous la juridiction de la Loi sur la protection de la jeunesse. La mesure prise en fonction de cette dernière loi n'est pas nécessairement reliée aux comportements sexuels déviants, et nous ne sommes pas en mesure d'identifier le type d'interventions suggérées en fonction de ces mêmes comportements.

Concernant la délinquance antérieure, vingt-six sujets n'en ont pas, vingt ont des antécédents de délinquance officiels et quatre ont des antécédents non-officiels révélés par le jeune lui-même. Plus de la moitié (13 sujets) ont commis plusieurs types de délits alors que 11 sujets n'ont commis qu'un seul type de délit. La plupart des actes illegaux commis sont des vols, des introductions par effractions, des voies de fait, des méfaits ou des bris. Seulement sept des 24 sujets qui possèdent des antécédents (officiels ou non-officiels) sont concernés par des délits de nature sexuelle.

Tous les sujets ayant êté trouvés coupables d'un délit sexuel avec pénétration ont obtenu une ordonnance de mise sous garde et aucun sujet ayant commis des attouchements n'a fait l'objet d'une telle mesure. La plupart de ces derniers ont obtenu une ordonnance de probation (20 sujets) ou une mesure de rechange (5 sujets). La majorité des sujets ayant fait usage de violence minimale $(17 / 21)$ ou moyenne $(9 / 12)$ ont obtenu une mesure de rechange ou une ordonnance de probation.

\section{B) Données liées à l'agir}

L'âge moyen des sujets lors de la commission du délit est de 14,8 ans. Le mode et la médiane sont de 15 ans. En fait, environ la moitié des sujets ont entre 14 et 15 ans inclusivement lors de la commission des gestes reprochés. 
L'échantillon est composé de 28 sujets dont l'écart d'âge est de cinq ans ou plus d'avec sa ou ses victimes (la moyenne d'écart d'âge étant alors de 9 ans et le mode de 11 ans), de 20 sujets qui ont agressé un pair ou un adulte (la moyenne d'écart d'âge étant alors de 0.65 et le mode de 1 an) et d'un sujet qui a agressé une ou des victimes des deux groupes d'âges. Il est impossible de préciser le type de délit en fonction de l'écart d'âge pour un sujet. puisque l'âge des victimes n'est pas précisé.

La nature des actes posés est de trois types: attouchements, tentative de pénétration et pénétration. Les attouchements regroupent les caresses des organes génitaux et les rapports bucco-génitaux (pour les fins de l'étude, les cas d'exhibitionnisme et de masturbation devant la victime ont été inclus sous cette rubrique). Les tentatives de pénétration regroupent les pénétrations de doigts dans le vagin ou l'anus et les tentatives de pénétration vaginale ou anale avec le sexe. Les pénétrations consistent en des pénétrations vaginales ou anales avec le sexe.

L'échantillon est composé de 37 sujets qui ont commis des attouchement, 5 sujets ayant commis des tentatives de pénétration et 6 sujets qui ont pénétré leur(s) victime(s). La nature des actes sexuels posés n'est pas précisée dans le dossier de deux sujets.

La violence utilisée lors du délit peut être divisée en plusieurs types : violence physique importante (coups, utilisation d'une arme), violence physique moyenne (contrainte physique), violence verbale (menaces, humiliations) et violence minimale (séduction, cadeaux, jeux). Quatre sujets ont fait usage de violence physique importante (jumelée parfois à une violence verbale), trois sujets ont fait usage uniquement de violence verbale, seize sujets ont utilisé une violence physique moyenne (jumelée parfois à une violence verbale) et 25 sujets ont utilisé une violence minimale. Le niveau de violence utilisé lors du délit n'est pas précisé dans deux dossiers de l'échantillon.

Concernant la présence de complices, 39 sujets étaient seuls lors de l'agir sexuel alors que onze sujets étaient avec un ou plusieurs complices : trois sujets avaient trois complices et huit sujets étaient accompagnés d'un seul complice.

\section{C) Données liées à la victime}

Les 50 sujets ont agressé un nombre total de 60 victimes. Huit sujets de l'échantillon ont fait plus d'une victime : sept sujets ont agressé chacun deux victimes et un sujet en a agressé quatre. Quarante-deux agresseurs n'ont qu'une seule victime. L'ensemble des victimes se répartit en 40 victimes de sexe féminin, 15 victimes de sexe masculin et 5 victimes dont le sexe n'est pas précisé. 
L'âge moyen des victimes est de 8.2 ans, le mode est de 4 ans et la médiane est de 7 ans. Environ $66 \%$ des victimes ont 8 ans et moins. On ne remarque aucune victime de sexe masculin âgée de douze ans et plus.

Une forte majorité de sujets (40/50) connaissaient leur(s) victimes(s) avant la commission du délit et seulement 10 sujets ne la ou les connaissaient pas.

Pour les besoins de la présente étude, deux catégories d'agressions sexuelles sont définies : l'agression sexuelle intrafamiliale est celle commise envers un membre de la fratrie biologique. alors que l'agression extrafamiliale est commise envers un membre de la parenté (cousine, neveu, etc.). un membre d'une même famille reconstituée ou un membre d'une famille d'accueil, ou encore envers quelqu'un qui n'a aucun lien de parenté avec l'agresseur.

Neuf sujets ( $18 \%$ ) ont commis un abus sexuel intrafamilial et quarante et un sujets $(82 \%)$ ont commis un abus sexuel extrafamilial. Parmi cette catégorie d'agressions sexuelles, six sujets sont les cousins de leur(s) victime(s) et quatre en sont les oncles, deux sujets font partie de la même famille d'accueil que leur(s) victime(s) et deux agresseurs et leur(s) victime(s) font partie d'une même famille reconstitué: pour cinq sujets, l'agression est survenue dans un contexte de gardiennage, trois sujets sont des voisins ou des connaissances de leur(s) victime(s), cinq sujets partagent une occupation avec leur(s) victime(s) (loisirs, école) et quatre sujets ont un contact personnalisé (amitié, amour, etc.) avec leur(s) victime(s). Finalement, dix adolescents n'avaient aucun lien avec la victime avant la commission du délit.

\section{ANALYSE DES DONNÉES}

À la suite de la première phase exploratoire de cette recherche, nous en sommes venus à faire certaines observations.

À partir de l'étude des 50 dossiers et des 10 entrevues. nous devons souligner l'absence d'uniformité des informations contenues dans les différentes évaluations : dans certains cas, on ne mentionne pas l'âge des victimes, la nature exacte des gestes posés, le niveau de violence utilisée. L’âge moyen des jeunes concernés était de 14,5 ans, et parmi eux, la moitié avaient des antécédents de délinquance autre que sexuelle, et sept sujets avaient des antécédents de délinquance sexuelle proprement dite. Pour $60 \%$ de l'ensemble de l'échantillon, l'écart d'âge entre l'agresseur et la victime était supérieur à 5 ans. Les victimes étaient majoritairement de sexe féminin, avec un âge moyen de 9 ans pour les 50 dossiers étudiés et 6 ans pour les sujets vus en entrevues. Quatre-vingt pout cent des sujets connaissaient leur victime et dans $60 \%$ de ces cas, il s'agissait d'attouchements et de 
caresses, et le niveau de violence utilisée était minimal : séduction, jeux. cadeaux. Ces résultats recoupent ceux déjà obtenus par d'autres chercheurs (Fehrenbach et al., 1986: Hornick, Bolitho et LeClaire, 1994).

Même si nous n'avons pas analysé nos données selon les critères utilisés par Jacob, McKibben et Proulx (1993), c'est-à-dire en subdivisant notre groupe en agresseurs contre les femmes et agresseurs contre les enfants, il est quand même possible de faire un certain nombre d'observations. Tout d'abord. en ce qui concerne la gravité des gestes posés pour $60 \%$ de notre population. il s'agissait uniquement d'attouchements. alors que pour la clientèle étudiée à l'Institut Pinel, aucun sujet (0/37) ne s'était limité à des attouchements : ceux-ci s'étaient livrés soit à une fellation, soit à une pénétration anale ou vaginale. Par ailleurs, dans l'échantillon de Jacob et al., un nombre plus élevé de sujets (41\% contre $13 \%)$ avaient des antécédents en matière de délinquance sexuelle. Enfin. les jeunes que nous avons interviewés semblent provenir de familles moins dysfonctionnelles que ceux du groupe étudié par Jacob et al. quant à la présence d'antécédents psychiatriques ou criminels dans la famille d'origine. On peut donc faire l'hypothèse, sans grand risque d'erreur, que les jeunes sont référés à l'Institut Pinel pour évaluation lorsque les comportements reprochés présentant une certaine gravité et que leur situation globale est plus complexe.

Ce type de comportement apparaît relativement tôt à l'adolescence (15 ans et moins) pour plus des deux tiers de nos sujets $(42 / 60)$ et pour un peu plus du tiers (23/60), il s'inscrit dans une carrière de délinquance polymorphe. Pour sept sujets, il s'agit d'une récidive de nature sexuelle. Le fait que les victimes soient très majoritairement de sexe féminin et en bas âge soulève un certain nombre de questions : est-ce un biais dû à notre échantillon, est-ce que l'on signale plus facilement aux autorités ces agressions que celles subies par des victimes de sexe masculin? Ces résultats correspondent toutefois à ceux déjà obtenus par les auteurs cités plus haut (Fehrenbach et al., 1986; Hornick et al., 1994).

Si l'on considère les mesures prises suite à la reconnaissance de culpabilité ou de la responsabilité en fonction du geste posé, on constate que, lorsqu'il y a eu pénétration ou tentative de pénétration. on avait recours à une mesure de garde en centre de réadaptation. Lorsqu'il s'agissait d'attouchements, on pouvait avoir recours à une mesure volontaire, à une mesure de rechange ou à une ordonnance de probation.

Au plan de la reconnaissance du délit, si on examine les 10 entrevues cliniques, seulement 4 sujets sont conscients du caractère délictuel du geste posé et reconnaissent avoir un problème à ce niveau. On peut souligner ici que 3 de ces 4 sujets avaient déjà participé à une thérapie pour jeunes abuseurs sexuels. Quant aux autres, il cationalisent ou minimisent l'importance du geste posé. Ils croyaient leur victime consentante au moment délit 
(ou même sollicitante dans certains cas). Si, après plus d'un an (temps écoulé entre la date de l'ordonnance et le moment de l'entrevue), $60 \%$ ne reconnaissent pas la gravité du geste posé ou ne sont pas conscients d'une problématique sexuelle, on peut faire l'hypothèse que l'action réadaptative aura eu peu d'impact sur cet aspect. On peut également en déduire que la seule intervention judiciaire n'est pas suffisante pour amorcer une prise de conscience à cet égard.

Les résultats aux tests psychométriques (matrice de Raven, échelle de Nowicki et Strickland sur le lieu de contrôle interne ou externe. échelle de Raskin et Hall sur la personnalité narcissique et échelle de Novaco sur le contrôle de la colère) ne présentent pas de différence significative entre les sujets ayant commis des attouchements et ceux ayant commis ou tenté de commettre un viol. Les sujets se situent généralement dans la normale pour leur performance aux quatre tests utilisés. Il est à noter que la majorité de ces jeunes ont bénéficié d'une intervention en centre de réadaptation, ce qui pourrait avoir eu pour effet de niveler les résultats.

$\mathrm{Au}$ Jesness, nos 10 sujets présentent des indices sérieux d'inadaptation (par exemple un score supérieur à 60 à l'échelle de mésadaptation sociale (SM) et à l'indice d'asocialité). Certains (8/10) obtiennent un score supérieur à 55 pour l'orientation aux valeurs (VO), 7/10 obtiennent un score supérieur à 55 pour l'agressivité manifeste (MA) et $6 / 10$ obtiennent un score supérieur à 55 pour l'autisme (AU). Il n'y a pas vraiment de profil spécifique permettant de distinguer ceux qui présentent des agirs pédophiliques et ceux qui présentent des agirs reliés au viol (pénétration et tentative de pénétration). On peut toutefois observer certaines tendances : les auteurs d'attouchements seraient plus immatures et auraient davantage tendance à refouler leurs sentiments de rébellion que les adolescents conventionnels. Les auteurs de viols, quant à eux, seraient davantage orientés vers les valeurs délinquantes, davantage portés à organiser la réalité en fonction de leur image de soi et exprimeraient plus facilement leur agressivité. On aurait pu s'attendre à des écarts plus importants entre ces deux groupes. mais cela pourrait tenir au petit nombre de sujets de notre échantillon.

Au plan social, la majorité de nos sujets $(6 / 10)$ ont vécu des difficultés relationnelles avec leurs parents, ont eu des difficultés au niveau scolaire, n'ont pas de réseau social (amis) minimal et ont été eux-mêmes victimes d'abus physiques ou sexuels.

\section{CONCLUSION}

À l'instar de Barbaree. Marshall et Hudson (1993), nous pourrions émettre l'hypothèse que les adolescents ayant commis une forme quelconque d'abus sexuel, forment un groupe hétérogène, tout comme les 
délinquants sexuels adultes. Selon eux, il n'existe toutefois pas, à l'heure actuelle, de typologie valable, basée sur une méthodologie rigoureuse et scientifique, permettant de distinguer certaines catégories mutuellement exclusives. Cet aspect de la recherche demeure donc à développer.

Pour en arriver là, toutefois, il nous apparaît prioritaire de sensibiliser les intervenants à la nécessité de recueillir systématiquement les informations de base mentionnées régulièrement dans la littérature, pour ensuite développer un protocole d'évaluation standardisé. permettant de poser un diagnostic différentiel et de faire un certain pronostic sur l'évolution future de chaque sujet pris individuellement, en fonction du niveau de risque qu'il représente. Par la suite, la décision de judiciariser ou non en vertu de la L.J.C. devrait tenir compte non seulement du niveau de risque que la personne présente. mais aussi de l'impact des procédures judiciaires tant sur l'adolescent que sur les victimes appelées à témoigner. À cet égard, Bala et Schwartz (1993) mentionnent que la poursuite en L.J.C. peut répondre à un objectif d'imputabilité en informant clairement le contrevenant que son geste est inacceptable socialement et qu'il doit être sanctionné. Par ailleurs, il semble exister une certaine controverse quant à l'aspect dissuasif d'une telle mesure, si la sanction n'est pas suivie d'un traitement approprié, comme le soulignent Schneider et Ervin (1988). Cette dernière observation nous amène à insister sur l'importance de développer des programmes d'intervention spécifiques à cette problématique particulière.

\section{BIBLIOGRAPHIE}

ABEL,G. G., MITTELMAN, M. S. et BECKER, J. V. (1985), "Sexual Offenders : Results of Assessment and Recommendations for Treatment ", in S. S. Ben-Aron, Webster et Hucker, (dir.) : Clinical Criminologv, Toronto. M. \& M. Graphics Ltd, pp. 191-206.

aUbUT, J., LAMONTAGNe, Y. (1980). Le traitement des déviants sexuels. Perspectives psychiatriques, $\mathrm{n}^{\mathrm{N}} 3$, pp. 1-12.

BALA, N., SCHWARTZ, I. (1993), "Leyal Responses (o) the Juvenile Sex Offender", in Barbaree. H.E., Marshall, W.L., Hudson, S.M. The Juvenile Sex Offender, Pp. $25-44$.

BARBAREE, H. E., MARSHALL, W. C. at HUDSON, S. M. (1993), The Juvenile Sex Offender, Londres, The Guilford Press. N.Y.

BECKER, J. V., KAPLAN, M. S., CUNNINGHAM-RATHNER, J. et KAVOUSSI, R. J. (1986), «Characteristics of Adotescent Incest Sexual Perpetrators : Preliminary Findings ", Journal of Fumily Violence, $\mathrm{n}^{\circ} 1$, pp. 85-97.

BELTRAMI. E. et al. (1979), «Ahus scxuel d'enfants : inceste père-fils comparé a la pédophilic homosexuelle", présentation laite au Sympositum " Enfince et Séxutlitể. Université du Quéhec à Montréal. 
BIGRAS, J. (1966), «Le sens moral et le masochisme dans l'inceste père-fille », Conadian Psychiatric Association Journal, vol. $2, \mathrm{n}^{\circ} 3$

BOISVERT, Y. (1989), Les Adolescents auteurs d'agressions sexuelles, présentation faite a la journée d'ćtude de Boscoville en mars 1989.

BOISVERT, Y. (1990), «Étude descriptive des adolescents violeurs et molesteurs d'enfants : identification des caractéristiques criminologiques, psychologiques et socio-familiales", travail dirigé, École de psycho-éducation.

BURGESS, A. W. (1978), Sexual Assault of Children and Adolescents, Lexington, Lexington Books.

CORMIER, B. et KENNEDY, M. (1962), «Psychodynamics of Father-Daughter Incest ", Canadian Psychiatric Association Journal, vol. 7, nº 5, pp. 203-217.

DOYON, P. (1989), Bilan de la situation relative aux abus chez la clientele desservie par les centres québécois de réadaptation pour jeunes en difficulté d'adaptation.

FEHRENBACK, P. A., SMITH, W., MONASTERSKY, C. et DEISHER, R. W. (1986), Adolescent Sexual Offenders : Offender and Offense Characteristics », American Journal of Orthopsychiatry, $\mathrm{n}^{\circ}$ 56, pp. 225-233.

FORGET, J. (1977), Validité prédictive de l'inventaire de Jesness et profil de l'adolescent de la Cour du Bien-être social, mémoire de man̂trise (Criminologie).

FRÉCHETTE, M. et LEBLANC, M. (1987), Délinquance et délinquants, Chicoutimi, Gaëtan Morin, éditeur.

FURR, K. D. et LANDRUS, G. (1971), «Raven Progressive Matrices Norms for Toronto, The Ontario Psychologist, vol. 3, $\mathrm{n}^{\circ} 3$, pp. 160-162.

GEBHARD, P. H. et al. (1965), Sex Offenders : An analysis of Types, Springfield, Charles $C$. Thomas.

GROTH, A. N. el BIRNBAUM, H. J. (1979), Men Who Rape: The Psychology of the Offender, New York, Plenum Press.

HORNICK, J. P., BOLITHO, F. H. et Le CLAIRE, D. (1994), Young Offenders and the Sexual Abuse of children, Ministère de la Justice, Canada, Technical Report.

JACOB, M., McKIBBEN, A. et PROULX, J. (1993), «Etude descriptive et comparative d'une population d'adolescents agresseurs sexuels", Criminologie, vol. 26, $\mathrm{n}^{\circ} 1$.

JESNESS, C. F. et The Jesness Inventory (édition révisée), Palo Alto, Consulting Psychologists Press.

jESNESS, C. F. (1983), WEDGE, R. F. (1984), «Validity of a Revised Jesness Inventory I Level Classification with Delinquents », Journal of Consulting and Clinical Psychology, vol. 52, pp. 997-1010.

JESNESS, C. F. (1986), "Validity of Jesness Inventory Classification with Delinquents », Educationnal and Psychological Measurements, vol. 46, pp. 947-961.

KEMPE, R. S. et KEMPE, C. H. (1978), Child Abuse, Cambridge, Mass., Harvard University Press.

KNIGHT, R. A. ef al., (1985), "Classification of Sexual Offenders: Perspectives, Mcthods and Validation ", in A.W. Burgess, (dir.), Rape and Sexual Assault, New York, Garland Publishing, pp. 222-293.

LEWIS, D. O. et al. (1979), «Juvenile Male Sexual Assaulters", American Journat of Psychiary, vol. 136. pp. 1194-1196. 
MAISONNEUVE, D. (1990), L'Agresseur sexuel adolescent, rapport de recherche. Expertise prédécisionnelle, Service des jeunes contrevenants CSSMM, $60 \mathrm{p}$.

MANSEAU, H. (1990), L'Abus sexuel et l'institutionnalisation de la protection de la jeunesse, Montréal, Presses de l'Université du Québec.

NOVACO, R. W. (1977), «Stress Inoculation : A Cognitive Therapy for Anger and its Application to a Case of Depression », Journal of Consuling and Clinical Psycholog. vol. 45, pp. 600-208.

NOWICKI, S. e1 STRICKLAND, B. (1973), «A Focus of Control Scale for Children», Joturnal of Consulting and Clinical Psvchology, vol. 40, no I, pp. 148-154.

RASKIN, R. et HALL, C. S. (1981), «The Narcissistic Personality Inventory : Alternate Form, Reliability and Further Evidence of Construct Validity, Journal of Personnality Assessment, vol. 45, $\mathrm{n}^{\circ}$ 2, pp. 159-162.

RAVEN, J. C. (1977), Manual for Raven's Progressive Matrices and Vocabulary Scales, Londres, H. K. Lewis Co. Ltd.

SCHNEIDER, A. L. et ERVIN, L. (1988), Deterrence and Juvenile Crime : Expanding the Behavioral Assumptions of Public Policy, article présenté à l'American Society of Criminology, Chicago.

SGROI, S. (1981), Handbook of Clinical Intenvention in Child Sexual Abuse, Lexing. ton, D.C. Heath and Compagny.

SHOOR, M. et al. (1962), "Syndrome of the Adolescent Child Molester", American Journal of Psychiatry, vol. 122, pp. 783-789.

SZABO, D. (1960), Contribution à l'étude de la délinquance sexuelle, Montréal, Fondation Richelieu.

VAN GIJSEGHEM, H. (1988), La Personnalité de l'abuseur sexuel, Montréal, Méridien. 